\title{
Unresolved Binary Exoplanet Host Stars Fit as Single Stars: Effects on the Stellar Parameters
}

\author{
E. Furlan ${ }^{1}$ (i) and S. B. Howell ${ }^{2}$ (i) \\ ${ }^{1}$ NASA Exoplanet Science Institute, Caltech/IPAC, Mail Code 100-22, 1200 E. California Blvd., Pasadena, CA 91125, USA; furlan@ipac.caltech.edu \\ 2 NASA Ames Research Center, Moffett Field, CA 94035, USA \\ Received 2020 March 10; revised 2020 June 1; accepted 2020 June 10; published 2020 July 22
}

\begin{abstract}
In this work, we quantify the effect of an unresolved companion star on the derived stellar parameters of the primary star if a blended spectrum is fit assuming the star is single. Fitting tools that determine stellar parameters from spectra typically fit for a single star, but we know that up to half of all exoplanet host stars may have one or more companion stars. We use high-resolution spectra of planet host stars in the Kepler field from the CaliforniaKepler Survey to create simulated binaries; we select eight stellar pairs and vary the contribution of the secondary star, then determine stellar parameters with SpecMatch-Emp and compare them to the parameters derived for the primary star alone. We find that, in most cases, the effective temperature, surface gravity, metallicity, and stellar radius derived from the composite spectrum are within $2-3 \sigma$ of the values determined from the unblended spectrum, but the deviations depend on the properties of the two stars. Relatively bright companion stars that are similar to the primary star have the largest effect on the derived parameters; in these cases, the stellar radii can be overestimated by up to $60 \%$. We find that metallicities are generally underestimated, with values up to eight times smaller than the typical uncertainty in $[\mathrm{Fe} / \mathrm{H}]$. Our study shows that follow-up observations are necessary to detect or set limits on stellar companions of planetary host stars so that stellar (and planet) parameters are as accurate as possible.
\end{abstract}

Unified Astronomy Thesaurus concepts: Stellar spectral lines (1630); Stellar properties (1624); Fundamental parameters of stars (555); High resolution spectroscopy (2096); Binary stars (154); Planet hosting stars (1242)

Supporting material: machine-readable table

\section{Introduction}

The discovery of an exoplanet orbiting its host star is the beginning of a process that aims at culminating with the determination of detailed properties of both the star and the planet. Only then can additional characterization work, such as transit spectroscopy or the assessment of potential habitability, be fully achieved. Over the last two decades, several missions and surveys have discovered many hundreds of exoplanets, most notably including the over 4700 confirmed planets and planet candidates discovered by the Kepler mission (Borucki 2016). Follow-up observations using space- and ground-based telescopes have provided imaging and spectroscopic details for the exoplanet-hosting stars (e.g., Howell et al. 2011; Adams et al. 2012, 2013; Lillo-Box et al. 2012, 2014; Dressing et al. 2014; Horch et al. 2014; Law et al. 2014; Marcy et al. 2014; Wang et al. 2014, 2015a, 2015b; Cartier et al. 2015; Everett et al. 2015; Gilliland et al. 2015; Torres et al. 2015; Baranec et al. 2016; Kraus et al. 2016; Furlan et al. 2017, 2018; Ziegler et al. 2017, 2018). Detailed follow-up continues for the planet candidates found by the Transiting Exoplanet Survey Satellite (TESS; Ricker et al. 2015), relying both on TESS team members as well as the community.

We have learned that the phrase "Know thy star, know thy planet" rings true, as the more accurately the host star parameters are known, the more definitively we can characterize any exoplanets it harbors. Transit observations, such as the ones carried out by Kepler and TESS, give us, in addition to some of the planet's orbital parameters, the exoplanet radius; however, it depends on the radius of the star it orbits and whether the photometric aperture is contaminated by "third light," i.e., unresolved stellar companions. Such a companion will dilute the transit, causing us to observe a shallower transit depth and leading us to derive a smaller planet radius (Ciardi et al. 2015). Horch et al. (2014) and Matson et al. (2018) have shown that approximately half of all exoplanet host stars may be binaries (or higher-order multiples), similar to the binary fraction observed in field stars (Raghavan et al. 2010). Other studies have shown that certain (mostly close) binaries are less likely to be planet host stars (Wang et al. 2014; Kraus et al. 2016). Moreover, about $30 \%$ of binaries in the solar neighborhood are comprised of about equal-mass stars (mass ratio $>0.8)$; for the closest binaries $(<100 \mathrm{au})$, this fraction increases to $\sim 40 \%$ (Raghavan et al. 2010). Such bright companions ( $\Delta m \lesssim 1$ for a Sun-like star), which would have the largest transit dilution effect, are thus fairly common. It is now well-accepted that, in order to determine the correct transit depth, one must correct for the third light, i.e., even perfect knowledge of the stellar radius is not enough. Without correction, the exoplanet radius and mean density will be incorrect (Furlan et al. 2017; Furlan \& Howell 2017; Hirsch et al. 2017; Teske et al. 2018) and calculations of the atmospheric scale height will be skewed (Batalha et al. 2017). In fact, this situation can be even more insidious because it is not always clear which star the exoplanet actually transits.

Corrections for a companion star (bound or line of sight) are now commonplace to adjust transit depths (e.g., Howell et al. 2019), but such corrections are not generally applied to spectral observations. Nearby, unresolved companions could result in incorrect stellar parameters, given that their spectral features are added to those of the primary star, but tools that extract stellar 
parameters from spectra typically fit for a single star (e.g., Torres et al. 2012; Endl \& Cochran 2016; Petigura et al. 2017).

Kolbl et al. (2015) did some work related to this idea by searching the large database of high-resolution spectra of the California Planet Search (CPS; Howard et al. 2010) for blended companion stars. They refit the high-resolution spectra of host stars of Kepler planet candidates, seeking to determine if a single star was most appropriate or if, in addition, a second star could be fit. They searched for spectral signatures of a companion star in the residual spectrum, after the best fit to the primary star had been subtracted. They found spectral evidence for companions in 63 sources (out of a sample of 1160 stars). Teske et al. (2015), using high-resolution imaging data, made an attempt to confirm the suspected companions in order to provide matches in these two techniques, allowing a better understanding of host systems. Unfortunately, no cases agreed between the two studies, leaving the situation of spectral decomposition and direct imaging confirmation a bit confused. The method of Kolbl et al. (2015) could be affected by incomplete line lists and certain differences in radial velocity (RV) and luminosity between the primary and companion star that make the detection of the companion unfeasible. However, this also suggests that the two techniques generally probe different populations of binaries, and also different binary separations, with just a small overlap in parameter space.

Stellar properties are determined accurately from highresolution spectroscopy (e.g., Torres et al. 2012; Mortier et al. 2013; Johnson et al. 2017; Petigura et al. 2017); the most commonly used techniques rely on model stellar atmospheres and atomic and molecular line lists. Some methods apply synthetic models to fit the spectral lines; these models are either precalculated to form a library (e.g., Buchhave et al. 2012; Petigura 2015; Endl \& Cochran 2016) or are synthesized to achieve a best fit to the observed spectrum (e.g., Valenti \& Piskunov 1996; Valenti \& Fischer 2005). Another method uses equivalent widths of Fe I and Fe II lines and compares them to model widths derived from model atmospheres, assuming LTE and excitation and ionization equilibrium (e.g., Sneden 1973; Santos et al. 2004; Mortier et al. 2013; Teixeira et al. 2016). For transiting planet host stars, the stellar densities can be determined directly from the transit light curve; through isochrone fits, the surface gravity of the star can then be derived (Sozzetti et al. 2007).

The stellar parameters of effective temperature $\left(T_{\text {eff }}\right)$, surface gravity $(\log (g))$, and metallicity $([\mathrm{Fe} / \mathrm{H}])$ are the observational values obtained, while fitting using stellar evolution models (such as the Dartmouth Stellar Evolution Program isochrones; Dotter et al. 2008) and/or asteroseismology leads to additional parameters such as radius, mass, and age. Combined with parallaxes from Gaia, stellar radii can be determined with a precision of $\sim 3 \%-8 \%$ (Berger et al. 2018; Fulton \& Petigura 2018, for the Kepler sample). Stellar radius and mass further constrain exoplanet parameters such as the planet radius and (combined with the orbital period) insolation flux and habitable zone inclusion. In addition, uncertainties in the stellar parameters, both from the data and the models used to derive them, contribute to the uncertainties of the planet parameters from the transit fit (for the planet radius) and RV fit (for the planet mass).

Using large samples, general statistical methods can be applied, as in Huber et al. (2013), to refine global stellar properties and check on overall correctness. For example,
Huber et al. (2013) took the pursuit of stellar parameters to a highly refined level by combining spectroscopic observations and asteroseismology to determine stellar radii to $\pm 3 \%$ and stellar masses to $\pm 7 \%$. Compared to just spectroscopically derived stellar parameters, which are more precise than those inferred from photometry and have typical uncertainties of $\sim 15 \%$ in stellar radius and $\sim 10 \%$ in stellar mass (Muirhead et al. 2012; Torres et al. 2012; Mortier et al. 2013; Huber et al. 2014; Johnson et al. 2017; Mathur et al. 2017), these are very accurate values and lead to more definitive exoplanet parameters. However, these types of analysis assume that the host star is single; if that is not the case, a spatially close companion star may make it more difficult to measure stellar oscillations (Sekaran et al. 2019) and can produce spectral contamination that will lessen the accuracy of the stellar fitting procedure's final values. Oscillations can also be changed by tidal interactions in close binaries, from being suppressed (Schonhut-Stasik et al. 2020) to being excited at specific frequencies (Fuller 2017).

Approximately half the stars are not single: for solar-type stars within $25 \mathrm{pc}$ of the Sun, about $45 \%$ have at least one companion star (Raghavan et al. 2010). For these nearby, multiple stellar systems, $11 \%$ of companions have periods less than 1000 days; this fraction increases to $21 \%$ and $40 \%$ for periods less than $10^{4}$ and $10^{5}$ days, respectively (Raghavan et al. 2010). Assuming a combined mass of $1.5 M_{\odot}$, a period of $10^{4}$ days corresponds to a semimajor axis of $\sim 10 \mathrm{au}$; projected on the sky, 10 au is less than $1^{\prime \prime}$ at distances beyond $10 \mathrm{pc}$, and so the binary system would likely be unresolved in seeinglimited imaging and spectroscopy. Considering that the peak of the period distribution of companions in the Raghavan et al. (2010) sample lies between $10^{3}$ and $10^{6}$ days, and many exoplanet host stars lie at distances of a few hundred pc, we expect most bound companions to exoplanet host stars to be unresolved in spectra obtained with $\sim 1^{\prime \prime}$-wide slits.

We seek to quantify the amount and type of additional error an undetected stellar companion might cause in the spectral fitting determination of stellar parameters. We have used a few high-resolution spectra of Kepler planet host stars, created blends, and fit these simulated binaries with SpecMatchEmp (Yee et al. 2017). We provide quantitative and qualitative estimates of how stellar blends affect the stellar parameters derived from these simulated spectra of unresolved stellar systems. For faint companions, the contamination is small and can be ignored. However, companions that are at least half as bright as the primary star lead to a complex contamination matrix of their influence on the determined stellar properties of $T_{\text {eff }}, \log (g)$, and $[\mathrm{Fe} / \mathrm{H}]$, resulting in values of these stellar parameters than can deviate up to $2-3 \sigma$ from the values derived from an unblended spectrum (where $\sigma$ is the uncertainty returned by the fitting code). In turn, these unaccounted-for deviations lessen the accuracy of the parameters determined for any orbiting exoplanet.

We describe the selection of the spectra analyzed for this study and our methodology to create blended spectra and derive their stellar parameters in Section 2, and the results of our stellar fits in Section 3. We then discuss the implications in Section 4, and give our conclusions in Section 5.

\section{Sample and Methodology}

We selected 16 stars from the California-Kepler Survey (CKS) to combine their spectra and create blended systems. As 
Table 1

Stellar Parameters Derived with SpecMatch-Emp for the Eight Stellar Pairs Used in This Work to Create Simulated Binaries

\begin{tabular}{|c|c|c|c|c|c|c|}
\hline KOI & $\bar{T} T_{\text {eff }}(\mathrm{K})$ & $\log (g)$ & {$[\mathrm{Fe} / \mathrm{H}]$} & $\overline{R_{*}\left(R_{\odot}\right)}$ & $\overline{\chi^{2}}$ & $\mathrm{~S} / \mathrm{N}$ \\
\hline \multicolumn{7}{|c|}{ Binary $1\left(\Delta T_{\text {eff }}=1404 \mathrm{~K}, L_{\mathrm{sec}} / L_{\text {prim }}=0.15\right)$} \\
\hline 2711 & $5882 \pm 110$ & $4.42 \pm 0.12$ & $0.08 \pm 0.09$ & $1.06 \pm 0.18$ & 1.95 & 53.0 \\
\hline 448 & $4478 \pm 70$ & $4.62 \pm 0.12$ & $0.04 \pm 0.09$ & $0.70 \pm 0.10$ & 6.51 & 29.8 \\
\hline \multicolumn{7}{|c|}{ Binary $2\left(\Delta T_{\text {eff }}=1218 \mathrm{~K}, L_{\mathrm{sec}} / L_{\text {prim }}=0.19\right)$} \\
\hline 692 & $5664 \pm 110$ & $4.25 \pm 0.12$ & $0.19 \pm 0.09$ & $1.06 \pm 0.18$ & 1.86 & 50.9 \\
\hline 1871 & $4446 \pm 70$ & $4.60 \pm 0.12$ & $0.20 \pm 0.09$ & $0.74 \pm 0.10$ & 7.96 & 25.8 \\
\hline \multicolumn{7}{|c|}{ Binary $3\left(\Delta T_{\text {eff }}=874 \mathrm{~K}, L_{\text {sec }} / L_{\text {prim }}=0.30\right)$} \\
\hline 2559 & $5791 \pm 110$ & $4.29 \pm 0.12$ & $0.10 \pm 0.09$ & $1.02 \pm 0.18$ & 2.18 & 48.4 \\
\hline 757 & $4917 \pm 110$ & $4.53 \pm 0.12$ & $0.10 \pm 0.09$ & $0.77 \pm 0.10$ & 8.59 & 24.4 \\
\hline \multicolumn{7}{|c|}{ Binary $4\left(\Delta T_{\text {eff }}=722 \mathrm{~K}, L_{\mathrm{sec}} / L_{\text {prim }}=0.43\right)$} \\
\hline 5622 & $5272 \pm 110$ & $4.55 \pm 0.12$ & $0.05 \pm 0.09$ & $0.82 \pm 0.10$ & 8.86 & 23.8 \\
\hline 870 & $4550 \pm 110$ & $4.57 \pm 0.12$ & $0.09 \pm 0.09$ & $0.72 \pm 0.10$ & 12.04 & 20.9 \\
\hline \multicolumn{7}{|c|}{ Binary $5\left(\Delta T_{\text {eff }}=363 \mathrm{~K}, L_{\mathrm{sec}} / L_{\text {prim }}=0.51\right)$} \\
\hline 1089 & $5736 \pm 110$ & $4.27 \pm 0.12$ & $0.08 \pm 0.09$ & $1.03 \pm 0.18$ & 7.10 & 28.0 \\
\hline 749 & $5373 \pm 110$ & $4.53 \pm 0.12$ & $0.05 \pm 0.09$ & $0.84 \pm 0.10$ & 3.73 & 37.6 \\
\hline \multicolumn{7}{|c|}{ Binary $6\left(\Delta T_{\text {eff }}=403 \mathrm{~K}, L_{\mathrm{sec}} / L_{\text {prim }}=0.61\right)$} \\
\hline 869 & $4989 \pm 110$ & $4.52 \pm 0.12$ & $0.13 \pm 0.09$ & $0.79 \pm 0.10$ & 11.50 & 21.4 \\
\hline 2339 & $4586 \pm 110$ & $4.54 \pm 0.12$ & $0.15 \pm 0.09$ & $0.73 \pm 0.10$ & 13.04 & 21.1 \\
\hline \multicolumn{7}{|c|}{ Binary $7\left(\Delta T_{\text {eff }}=132 \mathrm{~K}, L_{\mathrm{sec}} / L_{\text {prim }}=0.66\right)$} \\
\hline 116 & $5892 \pm 110$ & $4.37 \pm 0.12$ & $-0.11 \pm 0.09$ & $1.12 \pm 0.18$ & 0.43 & 135.3 \\
\hline 1379 & $5760 \pm 110$ & $4.49 \pm 0.12$ & $-0.11 \pm 0.09$ & $0.95 \pm 0.10$ & 2.90 & 45.4 \\
\hline \multicolumn{7}{|c|}{ Binary $8\left(\Delta T_{\text {eff }}=32 \mathrm{~K}, L_{\mathrm{sec}} / L_{\text {prim }}=0.84\right)$} \\
\hline 4072 & $5816 \pm 110$ & $4.22 \pm 0.12$ & $0.12 \pm 0.09$ & $1.20 \pm 0.18$ & 1.97 & 53.0 \\
\hline 3422 & $5784 \pm 110$ & $4.42 \pm 0.12$ & $0.12 \pm 0.09$ & $1.11 \pm 0.18$ & 1.70 & 57.3 \\
\hline
\end{tabular}

Notes. The first column lists the Kepler Object of Interest (KOI) number of the star. All stars host at least one planet candidate, with most stars (all except for KOI 1871,2559 , and 3422) hosting confirmed planets. The $\chi^{2}$ value is the median of the $\chi^{2}$ values returned by SpecMatch-Emp for each $100 \AA$ segment of the $5000-5800 \AA$ spectrum. The last column lists an estimate of the $\mathrm{S} / \mathrm{N}$ of the spectrum.

part of the CKS, 1305 stars in the Kepler field were observed with the High Resolution Echelle Spectrometer (HIRES; Vogt et al. 1994) on the Keck I telescope, with the goal of determining more accurate stellar parameters for Kepler planet host stars and thus for their transiting planets (Johnson et al. 2017; Petigura et al. 2017). As described in Petigura et al. (2017), two fitting codes, SpecMatch and SME@X-SEDE, were used to derive stellar parameters from the HIRES spectra of the 1305 stars in the CKS sample. The former code, which was actually developed for the CKS project to analyze Keck/ HIRES spectra (Petigura 2015), interpolates between model spectra to fit an observed spectrum. The latter code, which is based on Spectroscopy Made Easy (Valenti \& Piskunov 1996), calculates synthetic spectra via a radiative transfer code applied to model atmospheres. The stellar effective temperatures, surface gravities, and metallicities derived with these two methods agree very well, typically within the measurement uncertainties (Petigura et al. 2017; Brewer \& Fischer 2018). Their combined values were incorporated into a catalog. ${ }^{3}$

From the catalog of stellar parameters derived by the CKS team, we selected 16 targets to create blended spectra (see Table 1). In order to simulate spectra of unresolved binary stars consisting of a G-type star and a cooler companion, we selected dwarf stars with $T_{\text {eff }}$ in the $5000-6000 \mathrm{~K}$ range for the

\footnotetext{
3 Both the catalog and HIRES spectra from the CKS are available at https:// california-planet-search.github.io/cks-website/.
}

"primary" star and dwarf stars with $T_{\text {eff }}$ around $4400-5000 \mathrm{~K}$ range for the "secondary" star (with three exceptions, for which the companion star was chosen to be $<400 \mathrm{~K}$ cooler than the primary star). In addition, we also selected pairs of stars with similar metallicities (matching within $0.1 \mathrm{dex}$ ) to mimic binaries formed out of the same molecular cloud core and hence with the same initial composition. Multiplicity surveys of solar-type stars located within a few tens of parsecs from the Sun show that there is a roughly flat distribution in the mass ratios of the secondary and primary stars, with a small deficit for the lowest-mass companions (mass ratios $\lesssim 0.2$ ) and an overabundance of about equal-mass companions (Raghavan et al. 2010; Tokovinin 2014). Thus, our stellar pairs mimic binaries that are actually observed.

As a next step, we used the reduced HIRES spectra of our selected 16 stars from the CKS sample and determined their stellar parameters $\left(T_{\text {eff }}, \log (g),[\mathrm{Fe} / \mathrm{H}]\right.$, and $\left.R_{*}\right)$ using SpecMatch-Emp. ${ }^{4}$ This fitting code is a different version of SpecMatch (Yee et al. 2017). SpecMatch-Emp uses a library of observed spectra of calibrator stars to determine stellar parameters; the use of an "empirical" library results in more accurate fits for mid- to late- $\mathrm{K}$ and $\mathrm{M}$ stars (which are more difficult to fit with synthetic spectra, given numerous atomic and molecular lines with poorly known properties). The library contains 404 stars that were observed with

\footnotetext{
4 https://github.com/samuelyeewl/specmatch-emp
} 
Keck/HIRES as part of the CPS (Howard et al. 2010); these library stars have spectra with sufficiently high signal-to-noise (most have $\mathrm{S} / \mathrm{N}>100$ ) and well-determined stellar parameters from spectroscopy, spectrophotometry, interferometry, and asteroseismology (Yee et al. 2017). These parameters were retrieved from the literature; see Yee et al. (2017) for details. For stars without a complete set $\left(T_{\mathrm{eff}}, \log (g),[\mathrm{Fe} / \mathrm{H}], R_{*}, M_{*}\right)$, Yee et al. (2017) derived the missing parameters by fitting to the Dartmouth grid of stellar models (Dotter et al. 2008). The stellar parameters of the library stars cover $\sim 3000-7000 \mathrm{~K}$ in $T_{\text {eff, }} \sim 0.1-16 R_{\odot}$ in $R_{*}$, and -0.6 to $+0.6 \mathrm{dex}$ in $[\mathrm{Fe} / \mathrm{H}]$. The uncertainties returned by SpecMatch-Emp are set by the scatter of the differences between the stellar parameters derived by SpecMatch-Emp for the library stars and their library values. These uncertainties are smaller for cool stars, since their library parameters are more accurate.

Given that SpecMatch-Emp uses the 5000-5800 A range for its spectral fits, we only used the central (" $r$ ") HIRES spectrum (which covers 4975-6420 ̊). When fitting a spectrum with SpecMatch-Emp, it is first shifted onto the library wavelength scale (to account for the line-of-sight velocity of the target), then "matched" to the library spectra to find the best-matching spectra (which includes line broadening and normalization), and finally the parameters from the five best-matching spectra are combined by a weighted average to determine the stellar parameters (see Yee et al. 2017). To find the best-matching library spectra and then their best-fitting linear combination, an unnormalized $\chi^{2}$ statistic is used; to account for differences in continuum normalization, a cubic spline is fit to the residuals as $\chi^{2}$ is minimized. For both the matching and combination steps, the spectra are divided into $100 \AA$ segments and only wavelengths between 5000 and $5800 \AA$ are used. It would be possible to use a smaller range, but doing so might result in less reliable stellar parameters.

We derived the stellar parameters for our 16 targets using SpecMatch-Emp (see Table 1). In most cases, the derived stellar parameters agreed within the uncertainties with the parameters from the CKS catalog. Deviations of up to $3 \sigma$ in $T_{\text {eff }}$ were found for the coolest stars $\left(T_{\text {eff }} \sim 4400-4600 \mathrm{~K}\right)$, with the values derived with SpecMatch-Emp smaller by $\sim 120-180 \mathrm{~K}$. This may be expected, given that SpecMatch-Emp is more accurate for stars with $T_{\text {eff }} \lesssim 4500 \mathrm{~K}$ (Yee et al. 2017). For two of the cool stars (KOI 448 and 870), Muirhead et al. (2012) used a different method to determine effective temperatures by measuring spectral indices derived from $K$-band spectra and obtained even lower values (by $500-600 \mathrm{~K})$. However, this method becomes more uncertain for values larger than $\sim 3800 \mathrm{~K}$ (for details, see Muirhead et al. (2012)), and at least for KOI 870 the formal uncertainties imply that the derived $T_{\text {eff }}$ value agrees within $1 \sigma$ with the value derived with SpecMatch-Emp. Therefore, the $T_{\text {eff }}$ uncertainties for these cooler stars could be somewhat larger than their formally derived uncertainties, but likely not by more than a factor of two. In this work, we focus on the stellar parameters of the "primary" stars, which all have $T_{\text {eff }}$ values in the $5000-5900 \mathrm{~K}$ range, so even if the stellar parameters of cooler stars are in some cases more unreliable, they should not significantly affect the results of our fits. Moreover, we use the same fitting code for all spectra, so our results are selfconsistent and allow sensible comparisons.

We created simulated binary stars by combining the spectra of the pairs listed in Table 1. We started with the "shifted" version of the spectra, after SpecMatch-Emp has shifted the spectra onto the library wavelength scale. These eight pairs range from a difference in $T_{\text {eff }}$ of 1404 to just $32 \mathrm{~K}$ and a ratio in luminosity of the secondary relative to the primary star from 0.15 to 0.84 . Given that the reduced HIRES spectra are normalized, the spectrum of the companion star should be multiplied by a factor less than 1 before being added to the spectrum of the primary star in order to simulate a realistic binary companion. The luminosity ratio represents a rough approximation of the flux ratio in the optical (given differences in the spectral type and thus spectral energy distribution of our stellar pairs, we expect the optical flux ratios to be smaller for binaries with the coolest secondaries, ranging from about 0.6 to 0.7 of the luminosity ratios). We therefore scaled the companion stars by the luminosity ratio calculated from the effective temperatures and stellar radii given in Table 1 so they resemble bound secondaries.

Moreover, the signal-to-noise ratio $(\mathrm{S} / \mathrm{N})$ of the spectra varies (see Table 1), but in a bound system, where the combined spectrum is obtained, the contribution of the brighter star should have an $\mathrm{S} / \mathrm{N}$ greater than that of the fainter star. Thus, before adding the two spectra, we degraded the $\mathrm{S} / \mathrm{N}$ of one of the spectra by adding Gaussian noise to make sure that the ratio of the two S/Ns is roughly equal to the square root of the luminosity ratio (as a proxy for the brightness ratio in the optical). In most cases, the $\mathrm{S} / \mathrm{N}$ was degraded by less than $30 \%$ and so had just a minor effect on the spectrum. As noted by Yee et al. (2017), the SpecMatch-Emp algorithm is quite robust even at low $\mathrm{S} / \mathrm{N}$ (as low as 10); its accuracy is limited more by the matching process than by the noise in the spectrum.

After scaling and adjusting the $\mathrm{S} / \mathrm{N}$ of the spectra, we applied different RV shifts to the spectrum of the companion star, ranging from -500 to $+500 \mathrm{~km} \mathrm{~s}^{-1}$, to represent probable orbital motion of the two stars around the center of mass. Depending on the inclination angle of the binary system's orbit, the eccentricity and orbital period, and the mass ratio of the two stars, the semi-amplitude of the RVs of the secondary star $\left(K_{2}\right)$ can vary from just a few $\mathrm{km} \mathrm{s}^{-1}$ to several hundred $\mathrm{km} \mathrm{s}^{-1}$. For example, we estimated that, for binaries on circular orbits seen at intermediate inclination angles $\left(\sim 60^{\circ}\right)$ and with orbital periods between 100 and 3000 days, the $K_{2}$ values range from $\sim 10$ to $60 \mathrm{~km} \mathrm{~s}^{-1}$ (assuming mass ratios between 0.1 and 1.0); on orbits with shorter periods, $K_{2}$ values can reach over $250 \mathrm{~km} \mathrm{~s}^{-1}$, with even higher values for more edge-on orientations and smaller secondary masses. These $K_{2}$ values are also in agreement with observations (e.g., Tokovinin 2018). Thus, we expect the majority of $K_{2}$ values in a broad distribution over the $0-100 \mathrm{~km} \mathrm{~s}^{-1}$ range, with fewer values between 100 and $500 \mathrm{~km} \mathrm{~s}^{-1}$. Based on this motivation, we chose RV shifts of $\pm 500, \pm 400, \pm 300, \pm 200, \pm 100, \pm 90$, $\pm 80, \pm 70, \pm 60, \pm 50, \pm 40, \pm 30, \pm 20, \pm 10$, and also $0 \mathrm{~km} \mathrm{~s}^{-1}$ for the companion star's spectrum. The range of RV shifts we probed here are representative for close binaries, for which individual (i.e., spatially resolved) spectra are difficult to impossible to obtain.

Examples of the process of creating simulated binaries are shown in Figure 1, where we display the two individual spectra of a pair (after they have been shifted to the same wavelength frame and the cooler star scaled by the luminosity ratio of the two stars) and then various representative combinations of the 

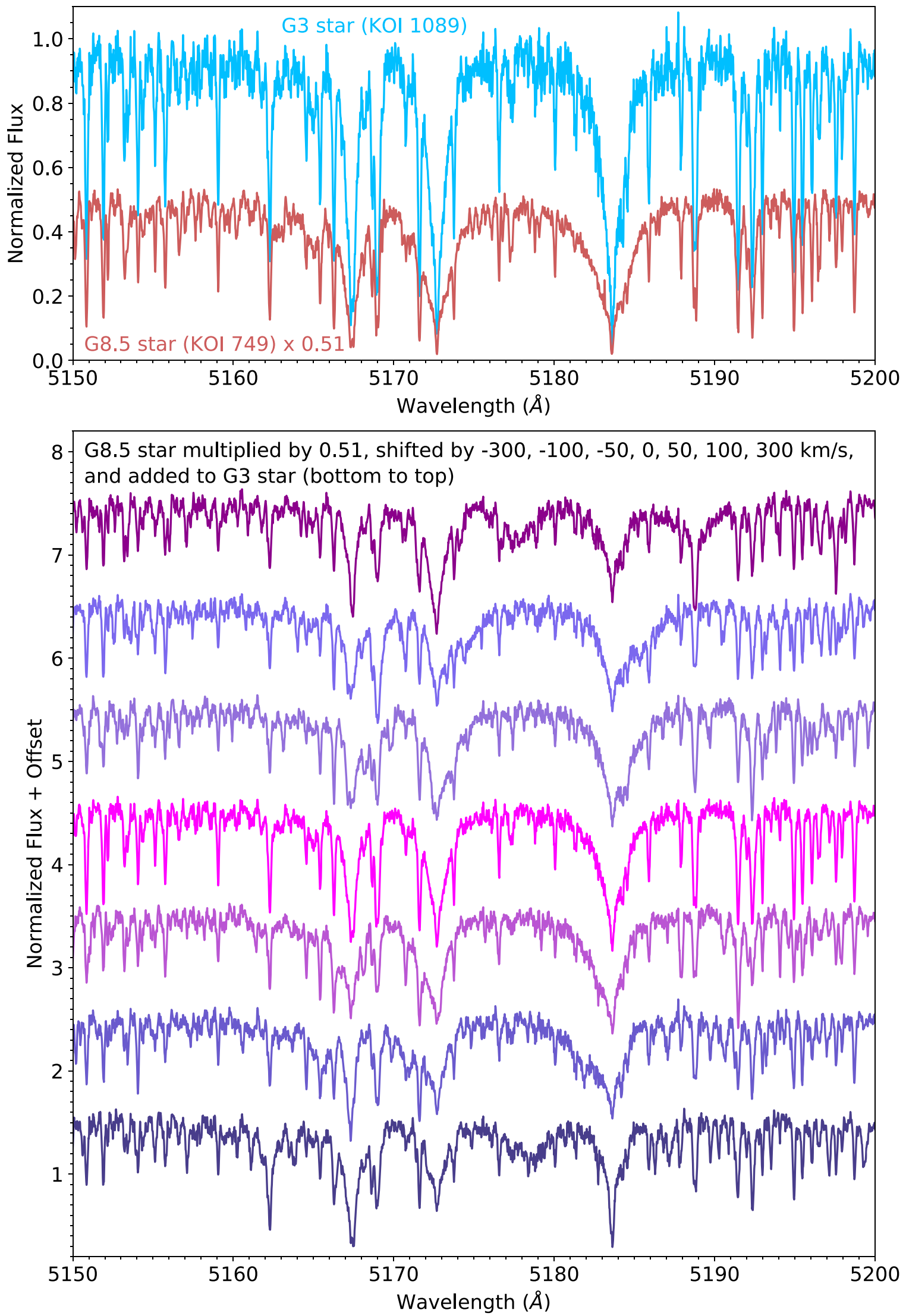

Figure 1. Combination of two CKS spectra to create a simulated binary (only the 5150-5200 A region is shown). Top panel displays the two spectra, where the spectrum of the cooler star has been multiplied by a factor equal to the luminosity ratio of the two stars. Bottom panel shows representative combinations of the two spectra after the multiplicative factor and RV shifts have been applied to the cooler star. 
Table 2

Stellar Parameters Derived with SpecMatch-Emp from the Blended Spectra of the 232 Simulated Binaries

\begin{tabular}{|c|c|c|c|c|c|c|c|}
\hline Star 1 & Star 2 & RV $\left(\mathrm{km} \mathrm{s}^{-1}\right)$ & $T_{\text {eff }}(\mathrm{K})$ & $\log (g)$ & {$[\mathrm{Fe} / \mathrm{H}]$} & $R_{*}\left(R_{\odot}\right)$ & $\overline{\chi^{2}}$ \\
\hline \multicolumn{8}{|c|}{ Binary $1\left(\Delta T_{\text {eff }}=1404 \mathrm{~K}, L_{\mathrm{sec}} / L_{\text {prim }}=0.15\right)$} \\
\hline KOI 2711 & KOI 0448 & -500 & $5956 \pm 110$ & $4.39 \pm 0.12$ & $-0.00 \pm 0.09$ & $1.11 \pm 0.18$ & 4.584 \\
\hline KOI 2711 & KOI 0448 & -400 & $5966 \pm 110$ & $4.38 \pm 0.12$ & $-0.02 \pm 0.09$ & $1.12 \pm 0.18$ & 4.407 \\
\hline KOI 2711 & KOI 0448 & -300 & $5986 \pm 110$ & $4.41 \pm 0.12$ & $0.00 \pm 0.09$ & $1.09 \pm 0.18$ & 4.312 \\
\hline KOI 2711 & KOI 0448 & -200 & $6000 \pm 110$ & $4.40 \pm 0.12$ & $0.02 \pm 0.09$ & $1.13 \pm 0.18$ & 4.351 \\
\hline KOI 2711 & KOI 0448 & -100 & $5968 \pm 110$ & $4.40 \pm 0.12$ & $-0.00 \pm 0.09$ & $1.11 \pm 0.18$ & 4.662 \\
\hline KOI 2711 & KOI 0448 & -90 & $5955 \pm 110$ & $4.38 \pm 0.12$ & $-0.02 \pm 0.09$ & $1.13 \pm 0.18$ & 4.526 \\
\hline KOI 2711 & KOI 0448 & -80 & $5970 \pm 110$ & $4.42 \pm 0.12$ & $-0.00 \pm 0.09$ & $1.08 \pm 0.18$ & 4.528 \\
\hline KOI 2711 & KOI 0448 & -70 & $5959 \pm 110$ & $4.42 \pm 0.12$ & $-0.01 \pm 0.09$ & $1.08 \pm 0.18$ & 4.580 \\
\hline KOI 2711 & KOI 0448 & -60 & $5965 \pm 110$ & $4.43 \pm 0.12$ & $-0.00 \pm 0.09$ & $1.08 \pm 0.18$ & 4.588 \\
\hline KOI 2711 & KOI 0448 & -50 & $5961 \pm 110$ & $4.44 \pm 0.12$ & $0.01 \pm 0.09$ & $1.07 \pm 0.18$ & 4.591 \\
\hline KOI 2711 & KOI 0448 & -40 & $5957 \pm 110$ & $4.44 \pm 0.12$ & $0.02 \pm 0.09$ & $1.07 \pm 0.18$ & 4.549 \\
\hline KOI 2711 & KOI 0448 & -30 & $5922 \pm 110$ & $4.46 \pm 0.12$ & $-0.01 \pm 0.09$ & $1.03 \pm 0.18$ & 4.466 \\
\hline KOI 2711 & KOI 0448 & -20 & $5894 \pm 110$ & $4.50 \pm 0.12$ & $-0.00 \pm 0.09$ & $1.00 \pm 0.10$ & 4.056 \\
\hline KOI 2711 & KOI 0448 & -10 & $5769 \pm 110$ & $4.51 \pm 0.12$ & $-0.01 \pm 0.09$ & $0.95 \pm 0.10$ & 2.899 \\
\hline KOI 2711 & KOI 0448 & 0 & $5601 \pm 110$ & $4.49 \pm 0.12$ & $-0.11 \pm 0.09$ & $0.92 \pm 0.10$ & 1.907 \\
\hline KOI 2711 & KOI 0448 & 10 & $5772 \pm 110$ & $4.51 \pm 0.12$ & $0.00 \pm 0.09$ & $0.95 \pm 0.10$ & 2.670 \\
\hline KOI 2711 & KOI 0448 & 20 & $5878 \pm 110$ & $4.50 \pm 0.12$ & $-0.02 \pm 0.09$ & $0.99 \pm 0.10$ & 4.018 \\
\hline KOI 2711 & KOI 0448 & 30 & $5939 \pm 110$ & $4.47 \pm 0.12$ & $0.02 \pm 0.09$ & $1.04 \pm 0.18$ & 4.511 \\
\hline KOI 2711 & KOI 0448 & 40 & $5950 \pm 110$ & $4.44 \pm 0.12$ & $0.01 \pm 0.09$ & $1.06 \pm 0.18$ & 4.681 \\
\hline KOI 2711 & KOI 0448 & 50 & $5953 \pm 110$ & $4.43 \pm 0.12$ & $-0.00 \pm 0.09$ & $1.07 \pm 0.18$ & 4.612 \\
\hline KOI 2711 & KOI 0448 & 60 & $5948 \pm 110$ & $4.41 \pm 0.12$ & $-0.02 \pm 0.09$ & $1.09 \pm 0.18$ & 4.630 \\
\hline KOI 2711 & KOI 0448 & 70 & $5922 \pm 110$ & $4.38 \pm 0.12$ & $-0.04 \pm 0.09$ & $1.13 \pm 0.18$ & 4.492 \\
\hline KOI 2711 & KOI 0448 & 80 & $5916 \pm 110$ & $4.37 \pm 0.12$ & $-0.04 \pm 0.09$ & $1.13 \pm 0.18$ & 4.489 \\
\hline KOI 2711 & KOI 0448 & 90 & $5943 \pm 110$ & $4.37 \pm 0.12$ & $-0.02 \pm 0.09$ & $1.13 \pm 0.18$ & 4.416 \\
\hline KOI 2711 & KOI 0448 & 100 & $5946 \pm 110$ & $4.36 \pm 0.12$ & $-0.02 \pm 0.09$ & $1.14 \pm 0.18$ & 4.490 \\
\hline KOI 2711 & KOI 0448 & 200 & $5977 \pm 110$ & $4.39 \pm 0.12$ & $0.02 \pm 0.09$ & $1.10 \pm 0.18$ & 4.478 \\
\hline KOI 2711 & KOI 0448 & 300 & $5946 \pm 110$ & $4.37 \pm 0.12$ & $-0.01 \pm 0.09$ & $1.12 \pm 0.18$ & 4.476 \\
\hline KOI 2711 & KOI 0448 & 400 & $5972 \pm 110$ & $4.36 \pm 0.12$ & $-0.01 \pm 0.09$ & $1.15 \pm 0.18$ & 4.412 \\
\hline KOI 2711 & KOI 0448 & 500 & $5952 \pm 110$ & $4.38 \pm 0.12$ & $-0.02 \pm 0.09$ & $1.12 \pm 0.18$ & 4.361 \\
\hline
\end{tabular}

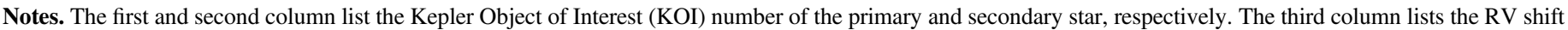

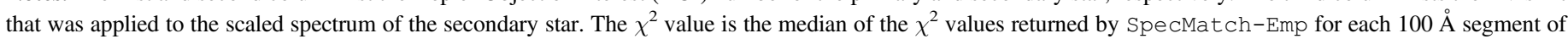
the 5000-5800 ̊ spectrum.

(This table is available in its entirety in machine-readable form.)

two spectra by applying an RV shift to the scaled spectrum of the cooler star before addition to the hotter star's spectrum.

\section{Results of Stellar Fits}

To quantify the effect of deriving stellar parameters from blended spectra, we used the eight pairs of spectra and created 29 simulated binaries for each pair: the spectrum of the cooler star was multiplied by the luminosity ratio of the two stars and also shifted in wavelength space by 29 different RV values (see Section 2) before co-adding it to the spectrum of the hotter star. After creating these blended spectra, we fit them with SpecMatch-Emp to derive $T_{\text {eff }}, \log (g),[\mathrm{Fe} / \mathrm{H}]$, and the stellar radius $\left(R_{*}\right)$. The results of the stellar fits for each simulated binary are listed in Table 2 and shown in Figures 2-5, where the difference between the stellar parameters derived from the blended spectrum and the parameters of the primary star are plotted as a function of RV shift applied to the secondary star.

The data points in these figures are color-coded based on the unnormalized $\chi^{2}$ value of the fit, which was calculated based on the output of SpecMatch-Emp. For the fits, the 5000-5800 $\AA$ spectra are divided into $100 \AA$ segments, and thus SpecMatch-Emp returns eight $\chi^{2}$ values for each fit. They are usually very similar, but to prevent outliers from inflating a $\chi^{2}$ value, we calculated the median of the eight $\chi^{2}$ values. Even when individual stars are fit, the $\chi^{2}$ value is not necessarily close to zero (see Table 1 ); values larger than $\sim 15$ likely suggest a bad fit. Therefore, stellar parameters from fits with large $\chi^{2}$ values are in general less reliable. It appears that, for most of the simulated binaries with a difference between effective temperatures of the two stars $\lesssim 750 \mathrm{~K}$ and RV shifts of the secondary star $\gtrsim 10 \mathrm{~km} \mathrm{~s}^{-1}$, the fits have large $\chi^{2}$ values.

For all simulated binaries, the discrepancies in derived stellar parameters are largest for the metallicities: the $[\mathrm{Fe} / \mathrm{H}]$ values tend to be smaller when derived from a blended spectrum by factors of a few relative to the values derived for the primary star alone, with the effect being larger the smaller the difference in luminosity between the primary and secondary star. Also, introducing RV shifts larger than $10 \mathrm{~km} \mathrm{~s}^{-1}$ to the spectrum of the second star results in a substantial decrease in derived $[\mathrm{Fe} / \mathrm{H}]$ values, but this trend plateaus beyond about $100 \mathrm{~km} \mathrm{~s}^{-1}$. Only zero RV shift or RV shifts up to $10 \mathrm{~km} \mathrm{~s}^{-1}$ will result in $[\mathrm{Fe} / \mathrm{H}]$ values consistent with values derived from an unblended spectrum of the primary star. However, for the brighter companion stars, the derived stellar parameters are also more unreliable, as gauged by the $\chi^{2}$ value returned by the fit.

The surface gravities and effective temperatures tend to agree within $\sim 5 \%$ with the values derived for the unblended primary star. Deviations in $T_{\text {eff }}$ are larger when zero or a very 

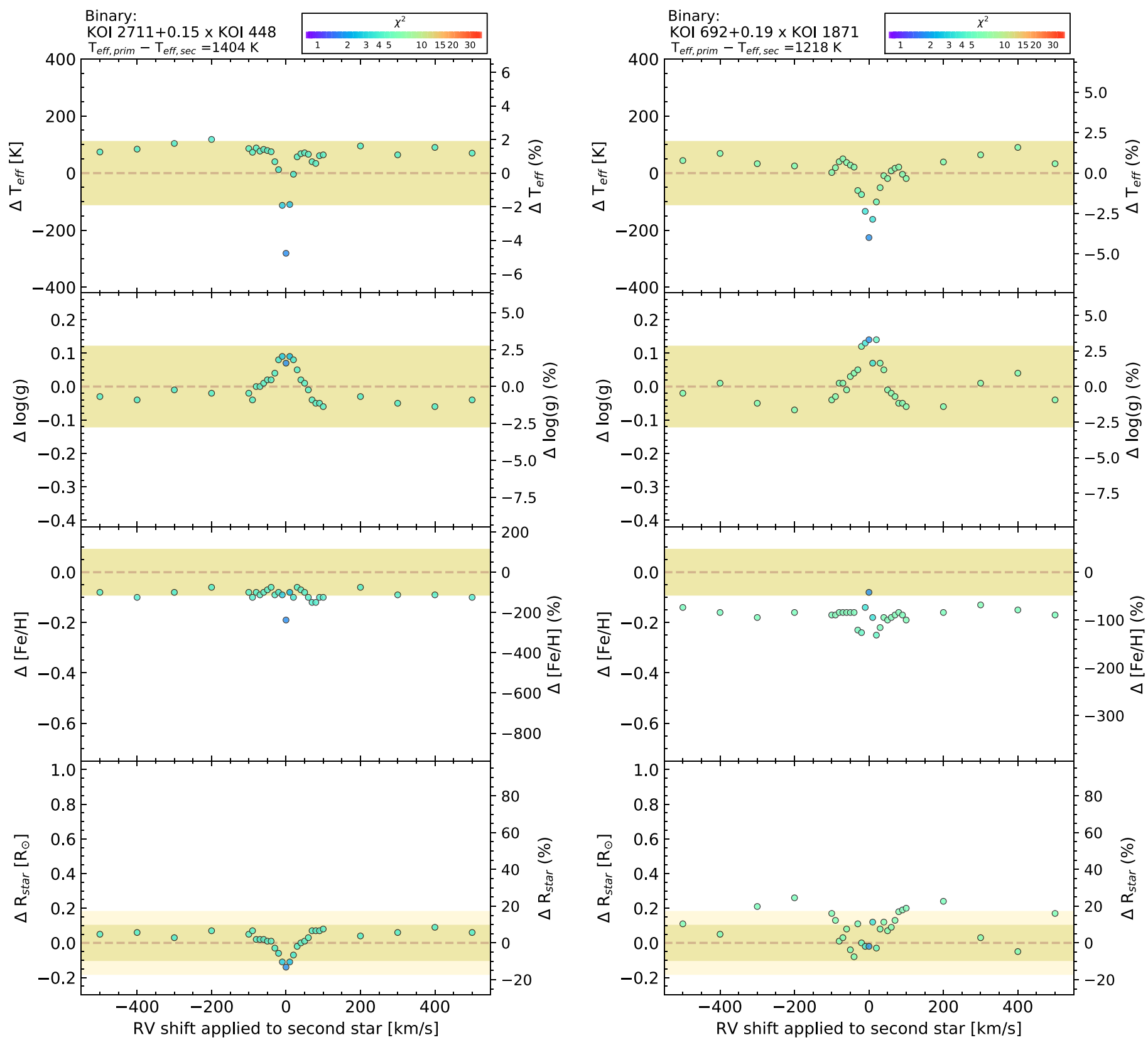

Figure 2. Results from fitting a blended spectrum created by adding a scaled and RV-shifted spectrum of a second (fainter) star to that of a primary star: differences in derived stellar parameters relative to the parameters of the primary star for Binary 1 (left) and Binary 2 (right) as a function of the RV shift applied to the second star. Plotting symbols are color-coded based on the $\chi^{2}$ value of the fit from SpecMatch-Emp (see legend). A positive value for a parameter difference means that the parameter derived from the blended spectrum is larger than the parameter derived from the spectrum of just the primary star. Conversely, a negative value means that the parameter derived from the unblended spectrum of the primary star is larger than the parameter derived from the blended spectrum. Shaded area delineates the uncertainty associated with the various parameters as returned by the SpecMatch-Emp fits. Uncertainty for $R_{*}$ can be either 0.1 or $0.18 R_{\odot}$, shown as darker and lighter shaded areas, respectively.

small RV shift is included for the companion, but only for the simulated binaries whose components have effective temperatures that differ by $\gtrsim 700 \mathrm{~K}$ (Binaries 1-4; see Table 1). In these cases, the effective temperature is underestimated for the blended spectrum by up to a few hundred $\mathrm{K}$; it is more in agreement with the value determined from the unblended spectrum when the companion spectrum is shifted by $>20 \mathrm{~km} \mathrm{~s}^{-1}$. The $\log (g)$ values do not show significant deviations for most binaries (the uncertainty returned by SpecMatch-Emp is 0.12); only some blended spectra cause $\log (g)$ values to be underestimated, but these values are also more unreliable.
The stellar radii derived from blended spectra agree quite well with those derived from the primary spectrum alone (considering typical uncertainties of $0.18 R_{\odot}$ from the fits), except for certain binaries when RV shifts are introduced. In such cases, the radii end up being overestimated, with typically larger values for larger RV shifts (up to $\sim 100 \mathrm{~km} \mathrm{~s}^{-1}$ ). However, as with the $\log (g)$ values, they are less reliable. At smaller RV shifts, stellar radii tend to be somewhat underestimated, but by less than the uncertainty of $0.18 R_{\text {Sun }}$ typically returned by SpecMatch-Emp.

In general, when the temperature difference between the primary and the companion star is $\lesssim 750 \mathrm{~K}$ (Binaries $4-8$ ), 

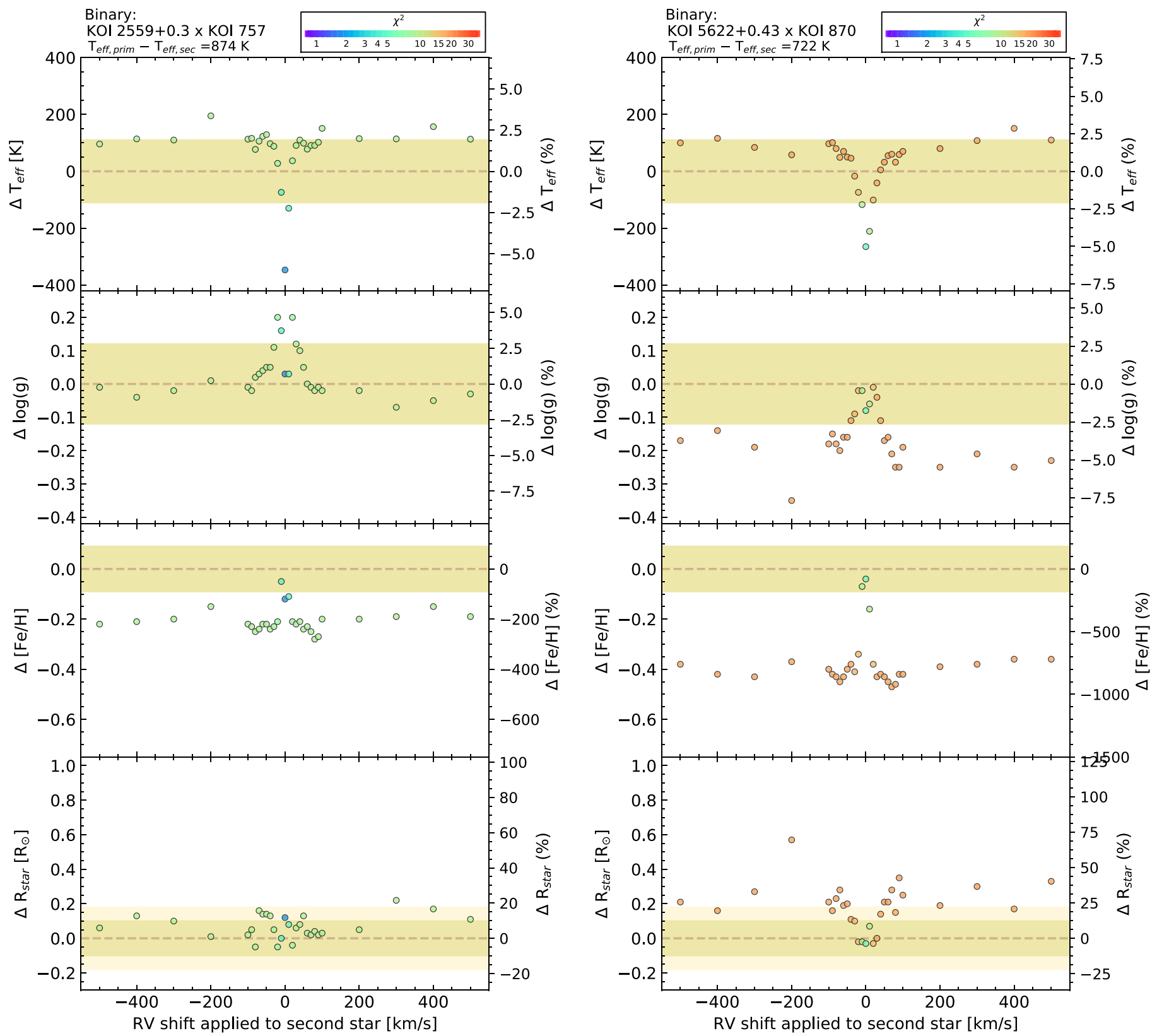

Figure 3. Similar to Figure 2, but for Binary 3 (left) and Binary 4 (right).

stellar parameters derived from blended spectra show more significant deviations when RV shifts greater than a few tens of $\mathrm{km} \mathrm{s}^{-1}$ are introduced. Effective temperatures are overestimated by up to $\sim 300 \mathrm{~K}, \log (g)$ values underestimated by up to $\sim 0.2-0.3 \mathrm{dex},[\mathrm{Fe} / \mathrm{H}]$ values are underestimated by $0.4-$ $0.7 \mathrm{dex}$, and $R_{*}$ is overestimated by up to $\sim 60 \%$.

Figure 6 combines the results shown in Figures 2-5; the median differences in parameter values are shown as a dasheddotted line. The large deviations in $T_{\text {eff }}$ for simulated binaries with no RV shift for the companion star are apparent, as well as the large underestimated $[\mathrm{Fe} / \mathrm{H}]$ values, which tend to be larger the brighter the companion star is (i.e., the smaller the difference in effective temperatures between the two stars). Metallicities, surface gravities, and stellar radii are least affected if no RV shift is present. Considering the uncertainties in the stellar parameters returned by the fit $\left(\sigma\left(T_{\text {eff }}\right)=110 \mathrm{~K}\right.$, $\left.\sigma(\log (g))=0.12, \sigma([\mathrm{Fe} / \mathrm{H}])=0.09, \quad \sigma\left(R_{\star}\right)=0.1-0.18 R_{\odot}\right)$, the $\log (g)$ values are overall least affected by adding a second stellar spectrum to that of the primary star. Median differences in parameter values for $\log (g)$ are within $1 \sigma$; those for $T_{\text {eff }}$ and $R_{*}$ are typically just larger than $1 \sigma$, while those for $[\mathrm{Fe} / \mathrm{H}]$ are at about $4 \sigma$. However, especially for binaries with brighter companion stars, $[\mathrm{Fe} / \mathrm{H}]$ can be underestimated by up to $8 \sigma$ and $R_{*}$ overestimated by up to $4 \sigma$.

From Figure 6, it is clear that stellar radii are significantly different only when the spectrum of a companion star is added with an RV shift of $\gtrsim 50 \mathrm{~km} \mathrm{~s}^{-1}$ and also a difference in $T_{\text {eff }}$ with respect to the primary of 720 or $\lesssim 400 \mathrm{~K}$. For smaller or zero RV shifts, and for simulated binaries with fainter secondaries, the stellar radius derived from the blended spectrum is not significantly different from the radius derived from the uncontaminated spectrum. However, the radius 

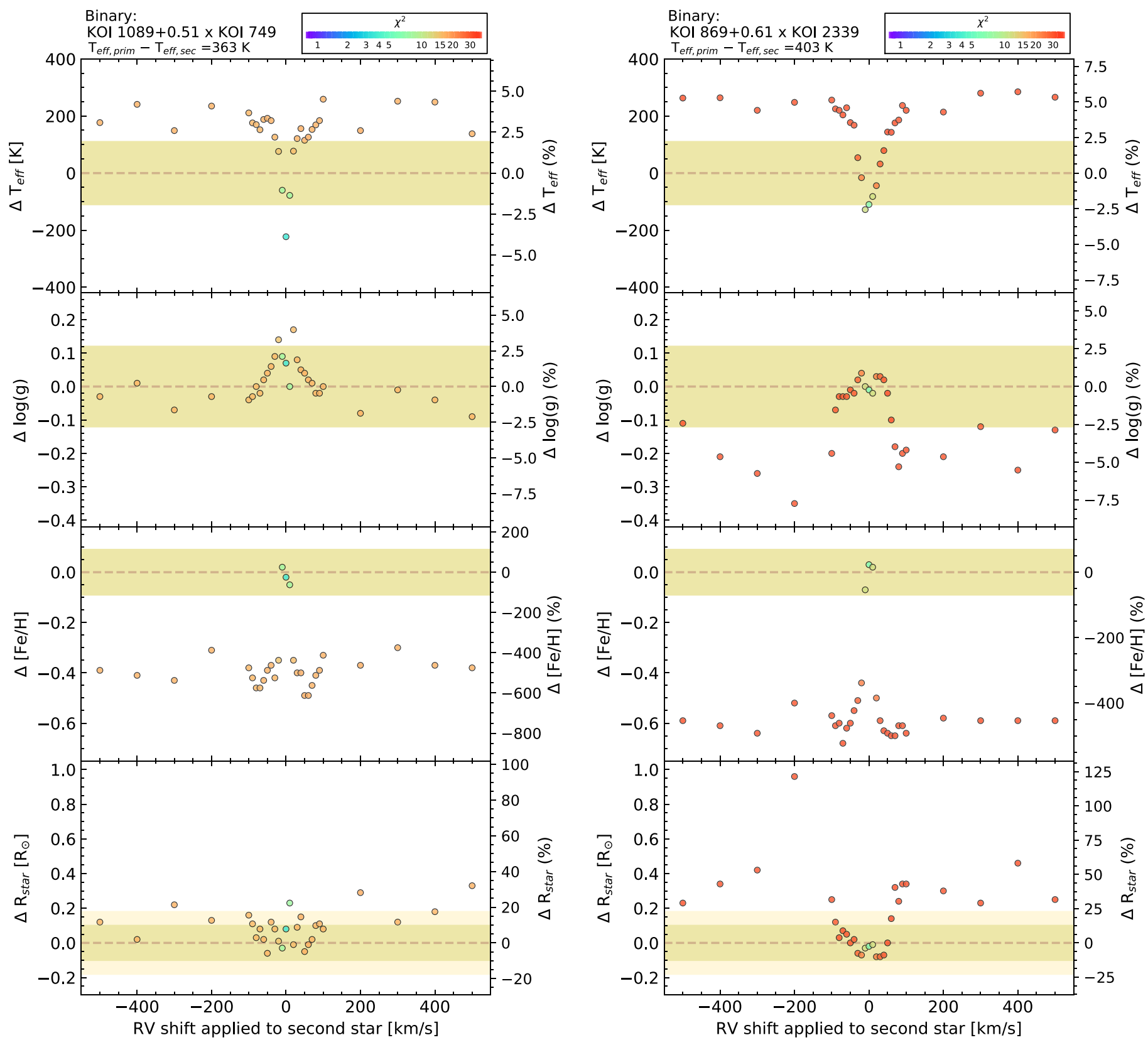

Figure 4. Similar to Figure 2, but for Binary 5 (left) and Binary 6 (right).

uncertainties as determined by SpecMatch-Emp are typically $0.18 R_{\odot}$ for the stars we selected as primaries (for stellar radii ranging from 0.8 to $1.2 R_{\odot}$, with a median value of $1.06 R_{\odot}$; see Table 1), placing them on the order of $17 \%$. These radii are just derived by comparing the observed spectrum to the library spectra and using a weighted average of the stellar radii of the five best-matching library stars. Thus, the radius uncertainties are set by the accuracy of the library parameters, and are relatively large. Other methods, like using isochrone fitting to convert $T_{\text {eff }}, \log (g)$, and $[\mathrm{Fe} / \mathrm{H}]$ to $M_{*}, R_{*}$, and age, can yield more precise stellar radii, with uncertainties of $\sim 11 \%$ (Johnson et al. 2017) or even 3\% (when Gaia parallaxes are used (Fulton \& Petigura 2018)). Using these methods, the effect of a blended companion star on the derived stellar radius might be more noticeable.

\section{Discussion}

Fitting a spectrum of a stellar blend with SpecMatch-Emp assuming only one star is present will result in stellar parameters that may be inaccurate, deviating by more than the $1 \sigma$ uncertainties returned by the fit (see Figure 7). If the companion star is faint, the effect on the derived stellar parameters of the primary star is very minor. At a minimum, the contaminating star will add some excess noise to the spectrum of the primary star. A brighter companion affects the determination of stellar parameters in nonintuitive ways - its effects depend on the properties of the two stars and how their spectral signatures are blended. The $\chi^{2}$ value returned by the fit can be used as an indicator of whether stellar parameters are still reliable, even though the largest $\chi^{2}$ values are not always associated with the largest discrepancies in derived stellar parameters. 

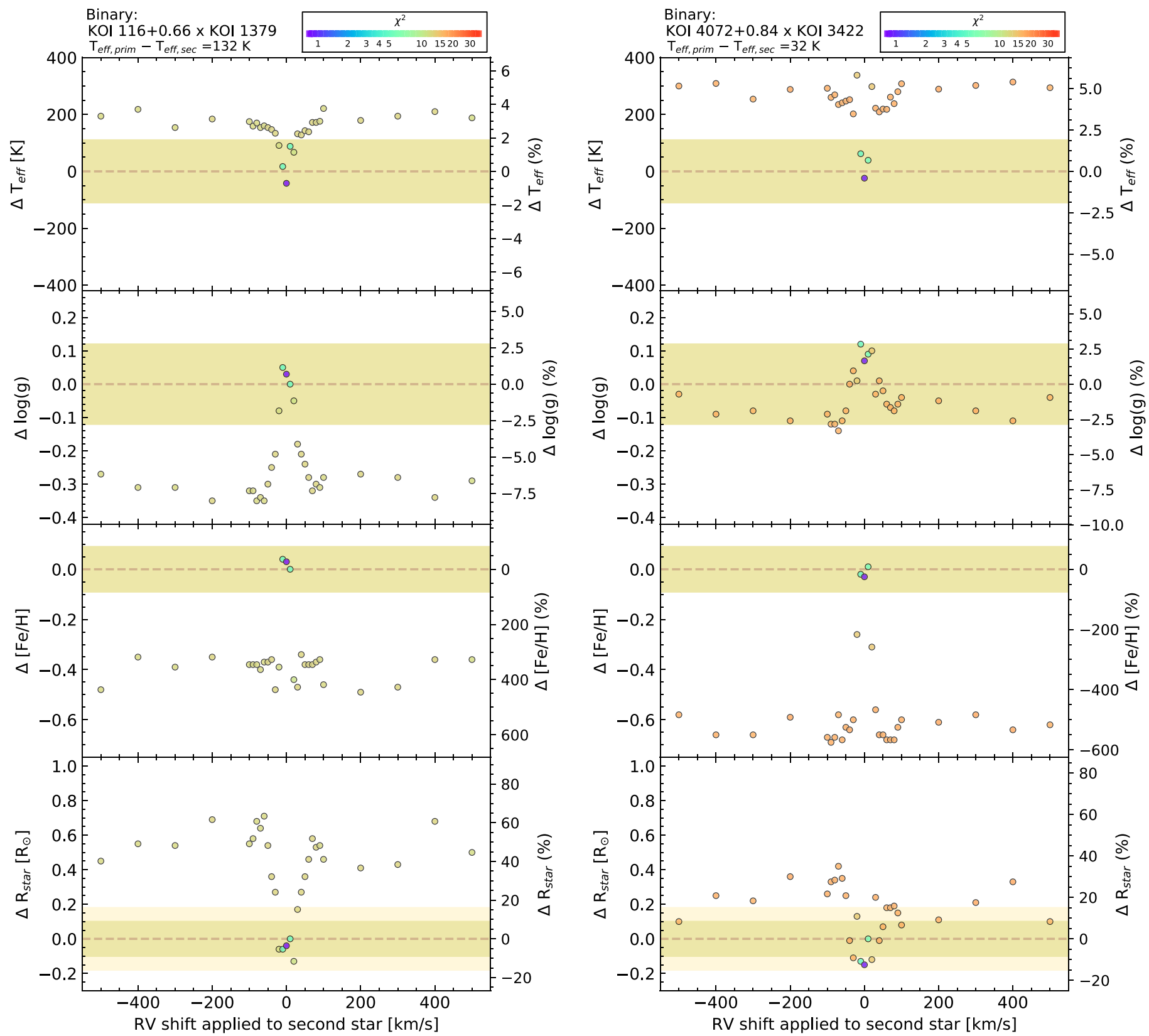

Figure 5. Similar to Figure 2, but for Binary 7 (left) and Binary 8 (right).

From our analysis of 232 simulated binaries, we find that, if the secondary star's spectrum is shifted in wavelength due to an $\mathrm{RV}$ difference with respect to the primary star, in some cases the stellar parameters of the primary star can still be retrieved reliably. It all depends whether SpecMatch-Emp can still determine a similar set of best-matching spectra as for the unblended spectrum of the primary star. While there are several line diagnostics that are sensitive to the various stellar parameters, such as ratios of weak metal lines (often of the same element and nearby in wavelength space) for $T_{\text {eff }}$, the $\mathrm{Mg}$ I b triplet for $\log (g)$, and various iron lines for $[\mathrm{Fe} / \mathrm{H}]($ e.g., Gray 1994, 1996; Sousa et al. 2020), it is difficult to determine which set of spectral lines most influences the outcome of SpecMatch-Emp. A few examples of how adding an RVshifted spectrum of a second star to that of the primary star affects some of the spectral lines are shown in Figure 8. The line profiles of the $\mathrm{Mg}$ I $\mathrm{b}$ triplet clearly change, and the depth of other metal lines is typically reduced. In general, weaker Mg I and Fe I lines would imply lower surface gravities and metallicities, respectively. It is also worth noting that altered line profiles, which would vary over time as the two stars orbit their center of mass, could affect the precision of $\mathrm{RV}$ measurements needed to measure exoplanet masses.

To see any correlations in how the various stellar parameters change due to a blended spectrum, in Figure 9 we compare the differences between $T_{\text {eff }}, \log (g),[\mathrm{Fe} / \mathrm{H}]$, and $R_{*}$ when these parameters are derived from a blended spectrum and when derived from the original spectrum of the primary star. When a blended spectrum results in $\operatorname{lower} \log (g)$ values, the derived $[\mathrm{Fe} / \mathrm{H}]$ values are also smaller, but the derived $T_{\text {eff }}$ values are typically larger. This mainly occurs when the luminosity ratio between secondary and primary star is $\gtrsim 0.4$ (or the temperature difference between primary and secondary star is $\lesssim 700 \mathrm{~K}$ ). When $T_{\text {eff }}$ values are underestimated, $[\mathrm{Fe} / \mathrm{H}]$ values are still 


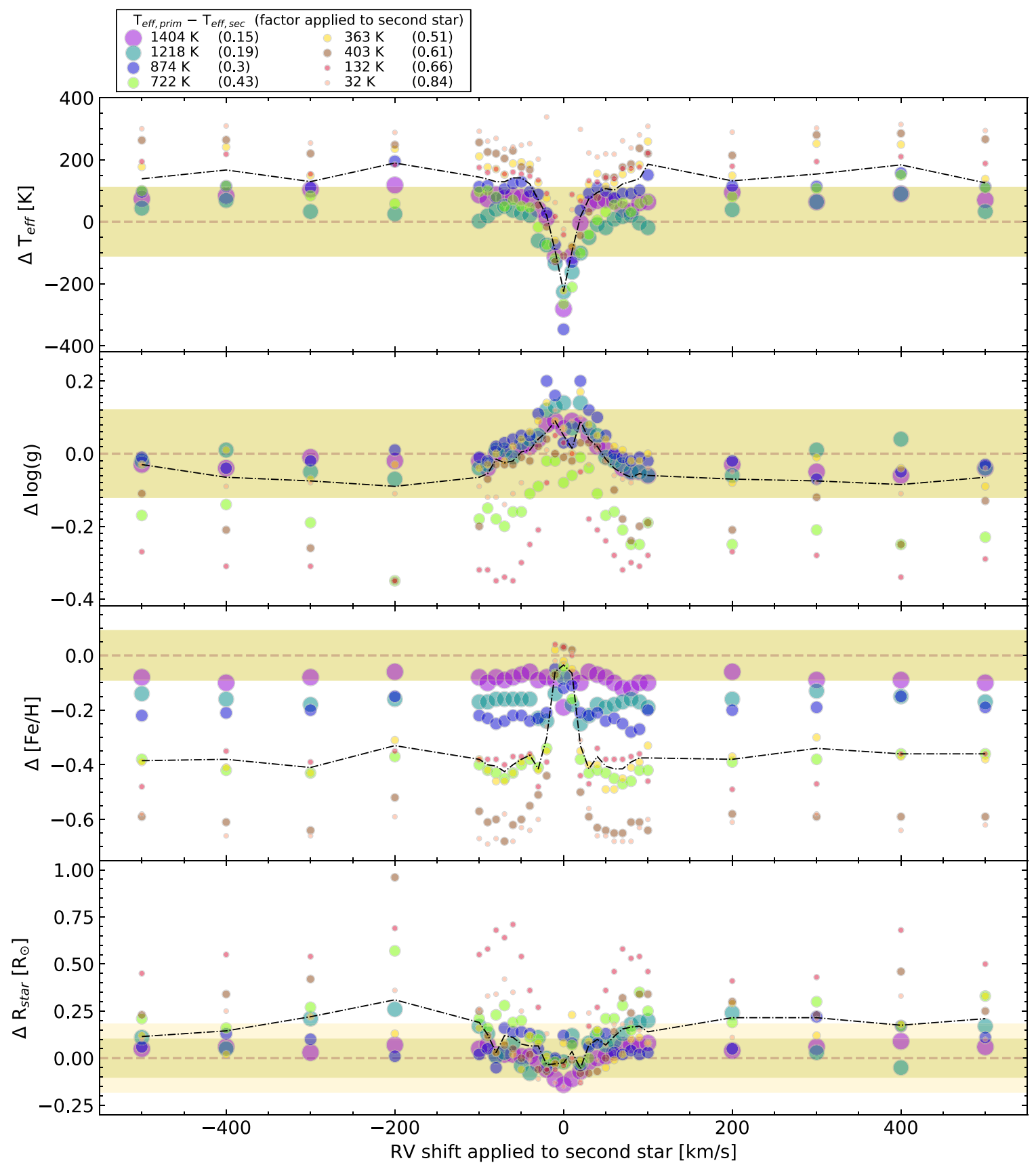

Figure 6. Results from Figures $2-5$ combined in one plot. Colors and sizes of plotting symbols vary with difference in $T_{\text {eff }}$ between primary and secondary star: larger symbols represent binaries with larger temperature differences between the two stars. Median differences in stellar parameters as a function of RV shift are shown with the black dashed-dotted line. Shaded areas have the same meaning as in Figures 2-5 (see caption of Figure 2).

underestimated, but by smaller amounts, and $\log (g)$ values are roughly in agreement with the values derived from the unblended spectrum. The differences in stellar radii are correlated with the differences in $\log (g)$, as expected $\left(\log (g) \propto R_{*}^{-2}\right)$; trends for the metallicity and effective temperature are less clear, except that the largest deviations in stellar radius occur when $T_{\text {eff }}$ is overestimated by $\sim 150-250 \mathrm{~K}$. This demonstrates that adding the spectrum of a second star above a certain brightness level will reduce the strength of lines of both pressure-sensitive and metallicity-sensitive lines, making stars appear to have lower surface gravity (or a larger radius) and lower metallicity. In these cases, the star's effective temperature is typically overestimated.
The primary star's effective temperature still agrees with the value derived from an unblended spectrum when the companion star is less than half as bright as the primary and its spectrum shifted by more than $\sim 20 \mathrm{~km} \mathrm{~s}^{-1}$. This is likely a result of temperature-sensitive lines of the primary star not being blended or distorted by the sufficiently shifted lines of the secondary star. If the two stars are very similar, only an RV shift of zero results in accurate parameters for the primary star (see Figure 6). In this case, the spectral features of the primary and secondary star overlap, and the fitting routine will still find an accurate match for the primary star. A blended companion star will cause the effective temperature of the primary star to not deviate more than $6 \%$, which is 

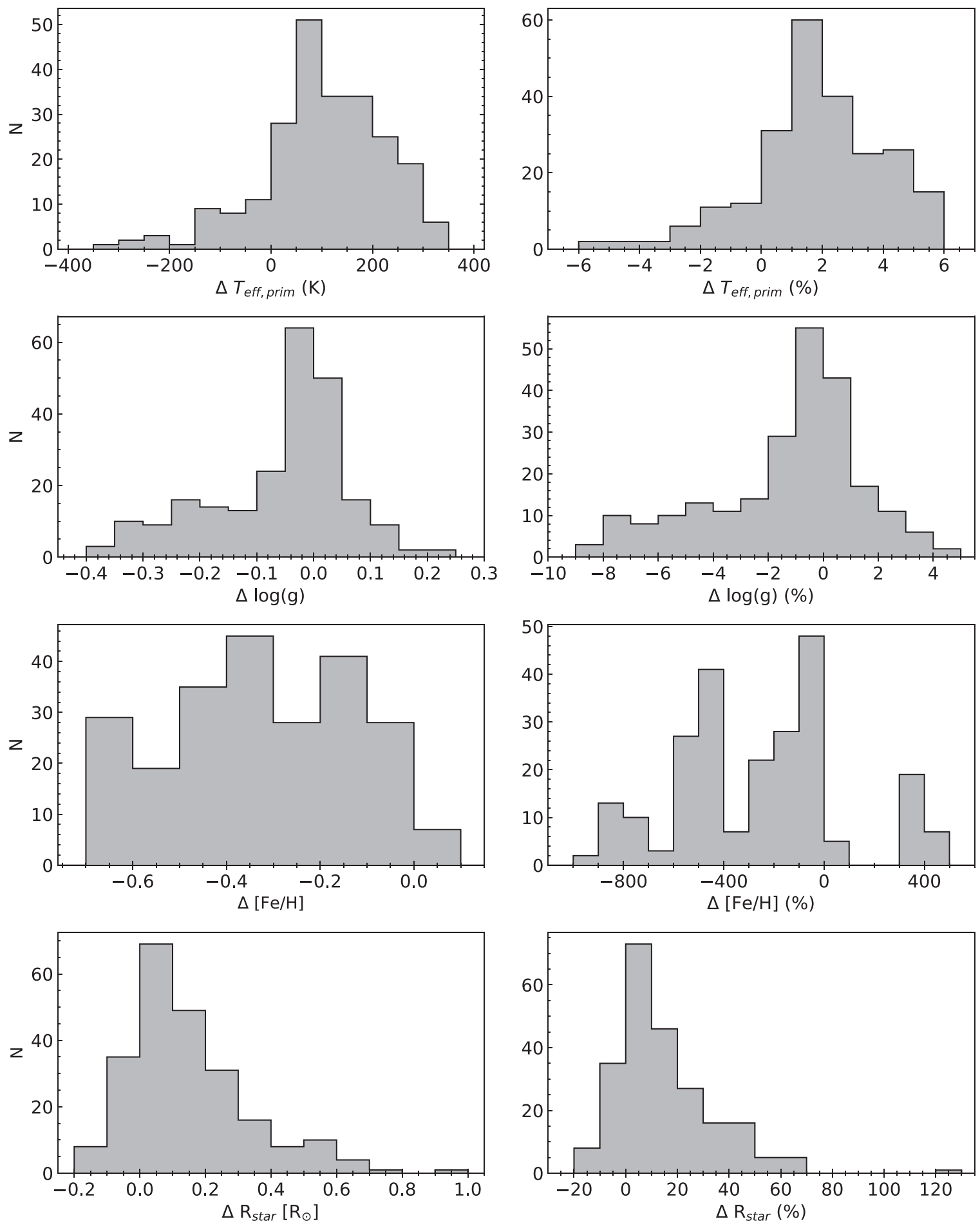

Figure 7. Histograms of the stellar parameter differences for the primary star (calculated as stellar parameters derived from a blended spectrum minus stellar parameters derived from the single spectrum; same data points as in Figures 2-5) for all 232 simulated binaries. Panels on the left show the differences in values; panels on the right show percentage differences (with respect to the value derived from the single spectrum of the primary star). In all panels, a value of zero means that the derived parameter is accurate for the primary star.

relatively small, but triple the $T_{\text {eff }}$ uncertainty returned by SpecMatch-Emp (see also Figure 7). This result is encouraging for studies that rely on temperature estimates from spectroscopy, such as the derivation of accurate $\log (g)$ and $R_{*}$ values from asteroseismology (e.g., Huber et al. 2013). The presence of a companion star will make the effective temperature more unreliable, but for most cases it is expected to lie within $2-3 \sigma$ of the actual value.
Surface gravities and stellar radii seem to be least affected when a blended spectrum is fit, especially when considering the respective $\sim 3 \%$ and $\sim 15 \%$ uncertainties in these parameters returned by SpecMatch-Emp. Fitting a blended spectrum results in deviations of $\log (g)$ and $R_{*}$ values of at most $\sim 2 \sigma$ in almost all cases (see also Figure 7). However, as mentioned earlier, different methods to derive $R_{*}$, like using isochrone fitting, yield more precise stellar radii, but they also rely on 

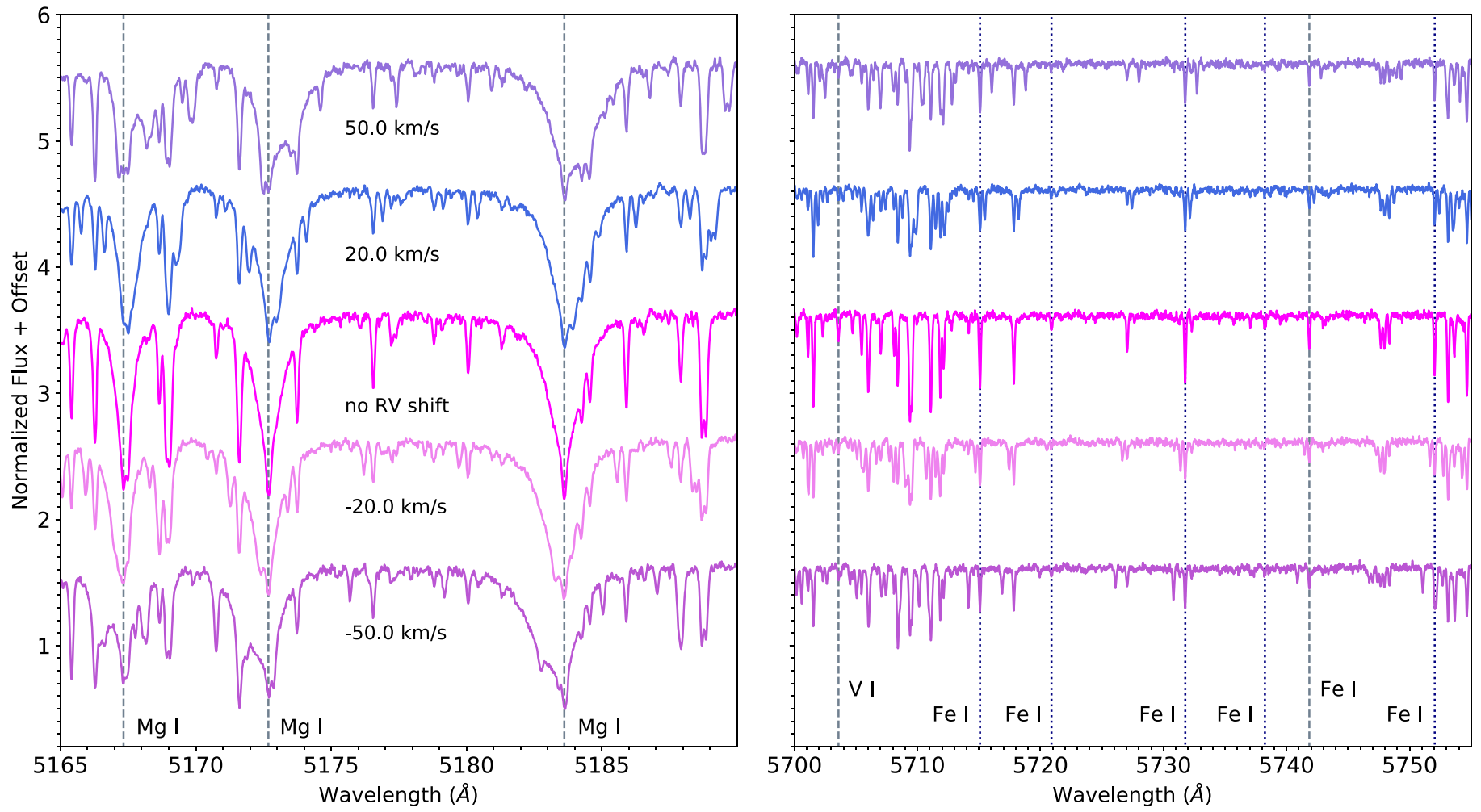

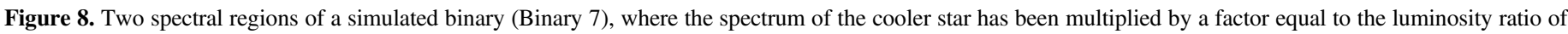

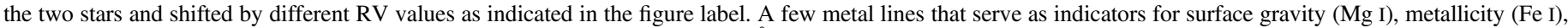
or effective temperature (e.g., ratio of V I and Fe I line at 5703.6 and $5741.9 \AA$, respectively) are also labeled.

other spectroscopically derived parameters. In this case, the uncertainties in $R_{*}$ would be dominated by the uncertainties in these parameters, in particular the effective temperature (see Fulton \& Petigura 2018). Thus, even if a precision of $3 \%$ could be achieved for $R_{*}$, a deviant $T_{\text {eff }}$ value due to a blended companion spectrum could increase the uncertainty of the stellar radius by several percentage points.

The stellar radius is an important parameter, especially for transiting planets, where the planet radius scales with the stellar radius. Any increase in the uncertainty of the stellar radius raises the uncertainty of the planet radius, thus affecting our interpretation of its properties, such as its density and atmospheric scale height, as well as the distribution of planet sizes (e.g., Weiss \& Marcy 2014; Rogers 2015; Fulton et al. 2017). Radius uncertainties of $\sim 10 \%$ or less are needed to see features and trends in planet radii distributions (Fulton et al. 2017; Fulton \& Petigura 2018). Planets transiting a star with an overestimated radius have derived planet radii that are too large as well (since $R_{\text {planet }} \propto R_{\star}$ ), and thus may be interpreted as having more volatiles than is actually the case. The stellar radius tends to be overestimated if a similar, bright star with $\mathrm{RV}$ shifts larger than a few tens of $\mathrm{km} \mathrm{s}^{-1}$ is blended with the primary star. Such a bright, nearby companion could be detected in high-resolution images or identified by its set of absorption lines in the blended spectrum; faint companions are more difficult to detect spectrally (becoming essentially undetectable once their luminosity ratio with respect to the primary drops below $\sim 10 \%$ ).

Metallicities are generally unreliable when derived from a stellar blend, even if the two stars have very similar metallicities. Unless the companion star is faint $(\sim 0.1$ the luminosity of the primary) or has zero or a very small RV shift relative to the primary, the metallicity derived from the combined spectrum is smaller by up to seven times the typical uncertainty in $[\mathrm{Fe} / \mathrm{H}]$ values for most cases. Indeed, once the $\mathrm{RV}$ shift is larger than about $50 \mathrm{~km} \mathrm{~s}^{-1}$ (which corresponds to shifts in wavelength of $\gtrsim 1 \AA$ ), the metallicity is underestimated by a constant amount; this results from the Fe I lines being diluted (and not distorted) by the light of the companion. Underestimating stellar metallicities could skew planet population studies, which have shown that the occurrence of large planets (from sub-Neptunes to Jupiters) increases with stellar metallicity (Santos et al. 2004; Fischer \& Valenti 2005; Johnson et al. 2010; Wang \& Fischer 2015; Narang et al. 2018; Petigura et al. 2018), while small, rocky planets $\left(R_{\mathrm{p}} \lesssim 1.7 R_{\oplus}\right)$ are found around stars with a wider range of metallicities (Buchhave et al. 2012; Everett et al. 2013; Wang \& Fischer 2015; Petigura et al. 2018). Higher actual metallicities for some stars could weaken the trend seen for some superEarths and sub-Neptunes. In addition, the metallicity of a star is used to inform planet formation models, where the metallicity of the protoplanetary disk is assumed to be the same as that of the star, and so more metal-rich stars are assumed to have disks with a higher solid surface density. In general, rocky cores of gas giant planets are thought to form more efficiently around metal-rich stars, thus allowing substantial atmospheres to be accreted (e.g., Dawson et al. 2015). Some models explaining the formation of super-Earths and sub-Neptunes might have to be adjusted if the metallicity of their host stars were underestimated. Finally, if certain stars are wrongly determined to be metal-poor, they may be seen as unlikely hosts for giant planets, so they could potentially be left out of target lists for planet searches or follow-up observations. 

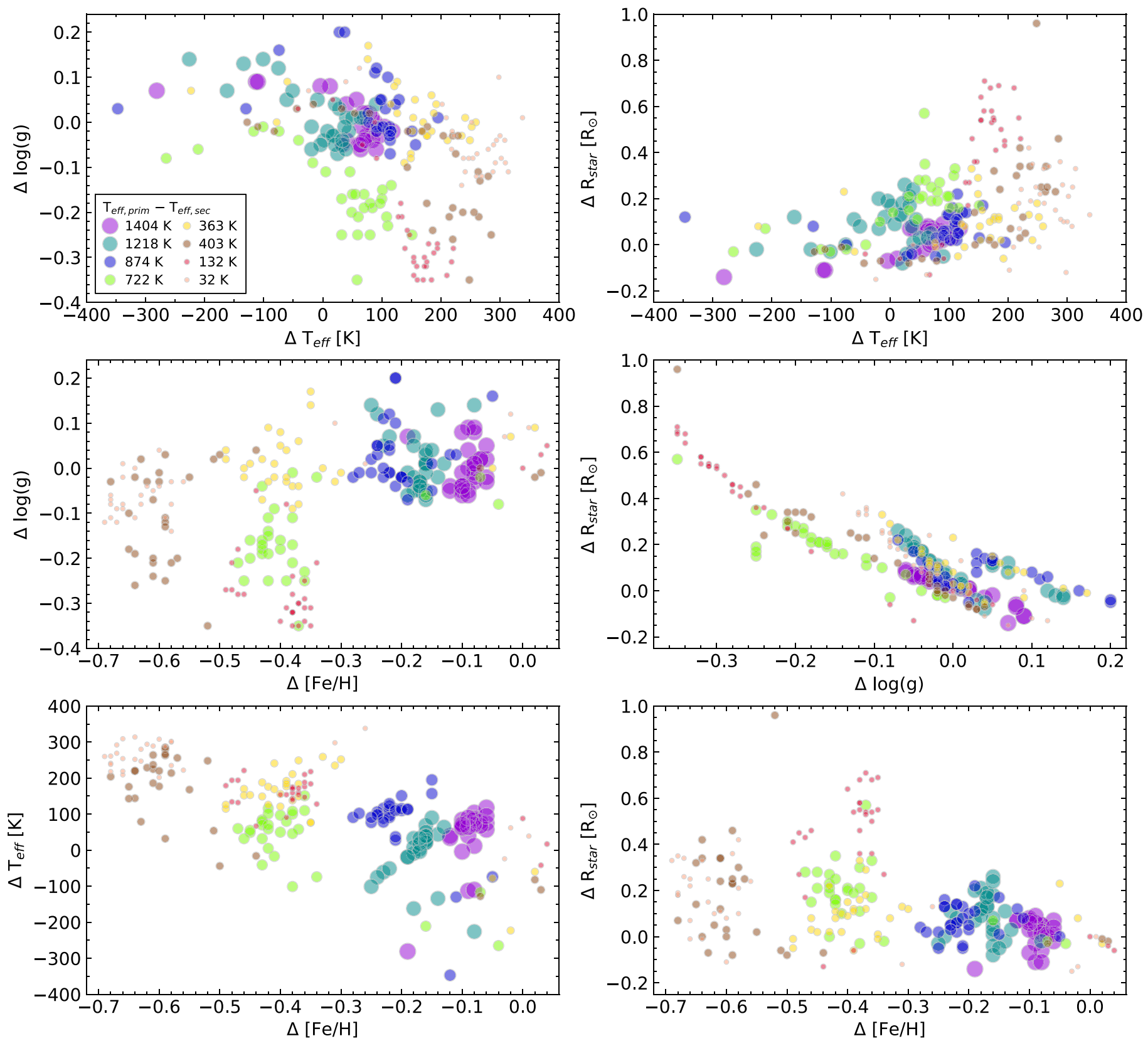

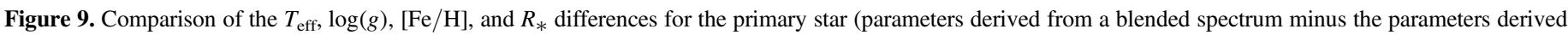

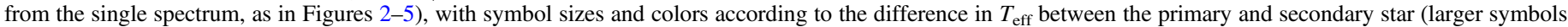
for larger temperature differences).

In summary, we note that the deviations introduced in the stellar parameters from fitting a blended spectrum strongly depend on the characteristics of this spectrum; the absorption lines from both stars, in particular whether the lines from the primary star are still identifiable by the fitting routine, as well as their S/N, will influence the outcome of the stellar fit. Based on the results of our 232 simulated binaries, we observed some trends, but there are also features that really depend on the two individual spectra that were combined. Thus, it is difficult to accurately predict for any specific case by how much the presence of a contaminating star will cause a deviation in the derived stellar parameters; in general, stellar parameters become more unreliable. In a bound binary system, the relative RV between the two stars will rarely be zero due to their orbital motion; when we observe such a system, it is more likely to be observed with some RV offset between the two components (expected to be a few tens of $\mathrm{km} \mathrm{s}^{-1}$ for binaries with separations of a few au). Additionally, repeat observations of close, bound pairs, such as those discovered by high-resolution imaging of nearby $\mathrm{K} 2$ and TESS exoplanet host stars, will exhibit changes in the companion star RV over time. Stellar parameters can thus be compared at different epochs and so determined more reliably. Accordingly, it is important to carry out follow-up, high-resolution imaging observations to search for close companions and vet planet candidates. Furthermore, with sufficiently high spectral resolution, the spectral lines of a close, relatively bright companion star that are shifted by tens of $\mathrm{km} \mathrm{s}^{-1}$ could be detected, thus alerting us to the presence of a spectroscopic binary. 


\section{Conclusions}

In this work, we have quantified how the stellar parameters are affected when they are derived from blended spectra. We have explored the contribution of a companion star to a blended spectrum by varying the type of the star (i.e., various differences in $T_{\text {eff }}$ between primary and secondary star) and its RV shift with respect to the primary. Typically, deviations in stellar parameters are up to $2-3 \sigma$ from the values derived from unblended spectra, with the effective temperatures and surface gravities least affected. Even stellar radii are not severely affected, given that their uncertainty (for unblended stars) is already of the order of $17 \%$ when derived with SpecMatchEmp. The exceptions are relatively bright companion stars that are almost as bright as and also similar in $T_{\text {eff }}$ to the primary star; these stars can cause an overestimation of the stellar radius by up to $\sim 60 \%$. We find that metallicities are very underestimated for all but the blends with the faintest companion stars; the metallicity is underestimated by an average of $\sim 4 \sigma$. These results are caused by the RV-shifted spectrum of the companion star being added to the spectrum of the primary star, which alters some absorption lines that are used as indicators for $T_{\text {eff }}, \log (g)$, and $[\mathrm{Fe} / \mathrm{H}]$. In addition, the modified line profiles could affect the precision of RV measurements used for the determination of planet masses. The $\chi^{2}$ of the fit is usually large when stellar parameters are significantly over- or underestimated, but there is not a linear correspondence between the $\chi^{2}$ value and the reliability of all parameters from a certain fit.

To account for the presence of a possible companion star, spectral fitting codes such as SpecMatch-Emp could be modified to include a second star in the fit. As was done in Kolbl et al. (2015), after fitting the spectrum of the primary star, a search for a second set of absorption lines could be performed on the residuals. However, this method works only for secondary stars that are sufficiently bright (even though Kolbl et al. (2015) claimed to be able to detect companions down to $1 \%$ of the total flux) and with a sufficient RV shift $\left(\sim 10 \mathrm{~km} \mathrm{~s}^{-1}\right)$ with respect to the primary star. Since it relies on subtracting the spectrum of the primary star and finding the secondary star in the residual spectrum, it is difficult to accurately determine the relative brightness of the secondary star and stellar parameters for secondaries that are either similar in spectral type or brightness to the primary star or are very faint (Kolbl et al. 2015). Therefore, ideally, spectral fitting codes could include an option to fit two stars simultaneously, even though it might become prohibitively expensive to carry out the computations (since the parameter space to explore is very large), and it might not always be clear whether a single or a binary star fit yields better results. More input from highresolution images would be needed to detect or place robust limits on possible companions that should be considered by the stellar fits.

To mitigate the effect of a contaminating star, obtaining a spectrum at different epochs (and thus different RV shifts between the two stars if dealing with a bound system) would yield more realistic uncertainties for the stellar parameters of the primary star. However, this is only feasible for binaries with periods less than a few decades, and it would also require sufficient spectral resolution to separate the lines of the two stars (especially once the RV shifts reach just a few km s${ }^{-1}$ ).

The only way to identify close, bound companion stars is by obtaining follow-up observations: high-resolution imaging to detect nearby, faint companions, and high-resolution spectroscopy over a sufficient time baseline to detect any spectral lines belonging to a companion. Of course, not all companions can be found, but particular attention should be paid to bright companions, which affect stellar fits the most. Given that planet parameters sensitively depend on stellar parameters, we should aim at determining the most accurate stellar parameters possible, particularly if we want to identify and characterize small, possibly habitable planets.

We thank Johanna Teske and David Ciardi for constructive feedback that improved the paper. We also thank the referee, whose comments made the discussion presented in this paper clearer and more thorough. Support for this work was provided by NASA through the NASA Exoplanet Exploration Program Office. This research has made use of NASA's Astrophysics Data System Bibliographic Services. It has also made use of the NASA Exoplanet Archive, which is operated by the California Institute of Technology, under contract with NASA under the Exoplanet Exploration Program.

\section{ORCID iDs}

E. Furlan (1) https://orcid.org/0000-0001-9800-6248

S. B. Howell (1) https://orcid.org/0000-0002-2532-2853

\section{References}

Adams, E. R., Ciardi, D. R., Dupree, A. K., et al. 2012, AJ, 144, 42 Adams, E. R., Dupree, A. K., Kulesa, C., \& McCarthy, D. 2013, AJ, 146, 9 Baranec, C., Ziegler, C., Law, N. M., et al. 2016, AJ, 152, 18

Batalha, N. E., Kempton, E. M.-R., \& Mbarek, R. 2017, ApJL, 836, L5 Berger, T. A., Huber, D., Gaidos, E., \& van Saders, J. L. 2018, ApJ, 866, 99 Borucki, W. J. 2016, RPPh, 79, 036901

Brewer, J. M., \& Fischer, D. A. 2018, ApJS, 237, 38

Buchhave, L. A., Latham, D. W., Johansen, A., et al. 2012, Natur, 486, 375 Cartier, K. M. S., Gilliland, R. L., Wright, J. T., \& Ciardi, D. R. 2015, ApJ, 804, 97

Ciardi, D. R., Beichman, C. A., Horch, E. P., \& Howell, S. B. 2015, ApJ, 805,16

Dawson, R. I., Chiang, E., \& Lee, E. J. 2015, MNRAS, 453, 1471

Dotter, A., Chaboyer, B., Jevremović, D., et al. 2008, ApJS, 178, 89

Dressing, C. D., Adams, E. R., Dupree, A. K., et al. 2014, AJ, 148, 78

Endl, M., \& Cochran, W. D. 2016, PASP, 128, 094502

Everett, M. E., Barclay, T., Ciardi, D. R., et al. 2015, AJ, 149, 55

Everett, M. E., Howell, S. B., Silva, D. R., \& Szkody, P. 2013, ApJ, 771, 107

Fischer, D. A., \& Valenti, J. 2005, ApJ, 622, 1102

Fuller, J. 2017, MNRAS, 472, 1538

Fulton, B. J., \& Petigura, E. A. 2018, AJ, 156, 264

Fulton, B. J., Petigura, E. A., Howard, A. W., et al. 2017, AJ, 154, 109

Furlan, E., Ciardi, D. R., Cochran, W. D., et al. 2018, ApJ, 861, 149

Furlan, E., Ciardi, D. R., Everett, M. E., et al. 2017, AJ, 153, 71

Furlan, E., \& Howell, S. B. 2017, AJ, 154, 66

Gilliland, R. L., Cartier, K. M. S., Adams, E. R., et al. 2015, AJ, 149, 24

Gray, D. F. 1994, PASP, 106, 1248

Gray, D. F. 1996, in IAU Symp. 176, Stellar Surface Structure, ed. K. G. Strassmeier \& J. L. Linsky (Dordrecht: Kluwer), 227

Hirsch, L. A., Ciardi, D. R., Howard, A. W., et al. 2017, AJ, 153, 117

Horch, E. P., Howell, S. B., Everett, M. E., \& Ciardi, D. R. 2014, ApJ, 795, 60 Howard, A. W., Johnson, J. A., Marcy, G. W., et al. 2010, ApJ, 721, 1467

Howell, S. B., Everett, M. E., Sherry, W., et al. 2011, AJ, 142, 19

Howell, S. B., Scott, N. J., Matson, R. A., et al. 2019, AJ, 158, 113

Huber, D., Chaplin, W. J., Christensen-Dalsgaard, J., et al. 2013, ApJ, 767, 127

Huber, D., Silva Aguirre, V., Matthews, J. M., et al. 2014, ApJS, 211, 2

Johnson, J. A., Aller, K. M., Howard, A. W., \& Crepp, J. R. 2010, PASP, 122,905

Johnson, J. A., Petigura, E. A., Fulton, B. J., et al. 2017, AJ, 154, 108

Kolbl, R., Marcy, G. W., Isaacson, H., \& Howard, A. W. 2015, AJ, 149, 18

Kraus, A. L., Ireland, M. J., Huber, D., et al. 2016, AJ, 152, 8

Law, N. M., Morton, T., Baranec, C., et al. 2014, ApJ, 791, 35

Lillo-Box, J., Barrado, D., \& Bouy, H. 2012, A\&A, 546, A10 
Lillo-Box, J., Barrado, D., \& Bouy, H. 2014, A\&A, 566, A103

Marcy, G. W., Isaacson, H., Howard, A. W., et al. 2014, ApJS, 210, 20

Mathur, S., Huber, D., Batalha, N. M., et al. 2017, ApJS, 229, 30

Matson, R. A., Howell, S. B., Horch, E. P., \& Everett, M. E. 2018, AJ, 156, 31

Mortier, A., Santos, N. C., Sousa, S. G., et al. 2013, A\&A, 558, A106

Muirhead, P. S., Hamren, K., Schlawin, E., et al. 2012, ApJS, 750, L37

Narang, M., Manoj, P., Furlan, E., et al. 2018, AJ, 156, 221

Petigura, E. A. 2015, PhD thesis, Univ. of California

Petigura, E. A., Howard, A. W., Marcy, G. W., et al. 2017, AJ, 154, 107

Petigura, E. A., Marcy, G. W., Winn, J. N., et al. 2018, AJ, 155, 89

Raghavan, D., McAllister, H. A., Henry, T. J., et al. 2010, ApJS, 190, 1

Ricker, G. R., Winn, J. N., Vanderspek, R., et al. 2015, JATIS, 1, 014003

Rogers, L. A. 2015, ApJ, 801, 41

Santos, N. C., Israelian, G., \& Mayor, M. 2004, A\&A, 415, 1153

Schonhut-Stasik, J., Huber, D., Baranec, C., et al. 2020, ApJ, 888, 34

Sekaran, S., Johnston, C., Tkachenko, A., et al. 2019, A\&A, 624, A140

Sneden, C. A. 1973, PhD thesis, Univ. Texas

Sousa, S. G., Alapini, A., Israelian, G., \& Santos, N. C. 2020, A\&A, 512, A13

Sozzetti, A., Torres, G., Charbonneeau, D., et al. 2007, ApJ, 664, 1190
Teixeira, G. D. C., Sousa, S. G., Tsantaki, M., et al. 2016, A\&A, 595, A15

Teske, J. K., Ciardi, D. R., Howell, S. B., et al. 2018, AJ, 156, 292

Teske, J. K., Everett, M. E., Hirsch, L., et al. 2015, AJ, 150, 144

Tokovinin, A. 2014, AJ, 147, 86

Tokovinin, A. 2018, ApJS, 235, 6

Torres, G., Fischer, D. A., Sozzetti, A., et al. 2012, ApJ, 757, 161

Torres, G., Kipping, D. M., Fressin, F., et al. 2015, ApJ, 800, 99

Valenti, J. A., \& Fischer, D. A. 2005, ApJS, 159, 141

Valenti, J. A., \& Piskunov, N. 1996, A\&AS, 118, 595

Vogt, S. S., Allen, S. L., Bigelow, B. C., et al. 1994, Proc. SPIE, 2198 362

Wang, J., \& Fischer, D. A. 2015, AJ, 149, 14

Wang, J., Fischer, D. A., Horch, E. P., \& Xie, J.-W. 2015a, ApJ, 806, 248

Wang, J., Fischer, D. A., Xie, J.-W., \& Ciardi, D. R. 2014, ApJ, 791, 111

Wang, J., Fischer, D. A., Xie, J.-W., \& Ciardi, D. R. 2015b, ApJ, 813, 130

Weiss, L. M., \& Marcy, G. W. 2014, ApJL, 783, L6

Yee, S. W., Petigura, E. A., \& von Braun, K. 2017, ApJ, 836, 77

Ziegler, C., Law, N. M., Baranec, C., et al. 2018, AJ, 155, 161

Ziegler, C., Law, N. M., Morton, T., et al. 2017, AJ, 153, 66 